\title{
CONHECIMENTO E UTILIZAÇĀ̉O DA FLORESTA PELOS ÍNDIOS WAIMIRI-ATROARI DO RIO CAMANAU - AMAZONAS (1)
}

\author{
Robert P. Miller (2) \\ Elisa V. Wandelli (3) \\ Pierre Grenand (4)
}

\begin{abstract}
RESUMO - Os índios Waimiri Atroari têm hábitos e costumes bastantes intactos e retiram da floresta, roça e rio os materiais necessários para a alimentação, utensílios e moradia. Este trabalho objetivou fazer um levantamento preliminar do conhecimento e utilização da floresta pelos índios. Foram inventariados todas as árvores com DAP $>6 \mathrm{~cm}$ em cinco parcelas de $10 \times 20 \mathrm{~m}$, localizadas $0.5 \mathrm{~km}$ da aldeia, ao longo de um transecto abrangendo um gradiente de terra firme - baixio. Foram coletadas amostras para herbário e anotadas informações sobre uso e nome indigena. Em 0.1 hectare foram encontradas 135 árvores de 60 espécies, sendo as famílias mais comuns: Lecythidaceae, Leguminosae sensu latu, Burseraceae, Bombacaceae e Lauraceae. Noventa e cinco por cento dessas espécies eram identificadas com um nome específico na língua Waimiri Atroari e as demais receberam um nome genérico ("wiwe ') que quer dizer árvore. De uma amostra de 34 espécies sessenta e cinco por cento tinham algum uso específico. Estes dados demonstram um alto grau de conhecimento e utilização da floresta pelos Waimiri Atroari.
\end{abstract}

Palavras Chaves: Waimiri Atroari, etnobotânica, florística, Amazônia.

\begin{abstract}
The Waimiri Atroari retain much of their original culture and take from the forest, river, and plantations their food and most of the materials necessary for construction, craft, and medicines. This study attempted to quantify their knowledge and use of forest. Five quadrats of $10 \times 20 \mathrm{~m}$ (total $=0,1$ hectare) were located along a transition from "terra firme" (upland) to "baixio" (stream valley). Herbarium specimens were collected from trees above $6 \mathrm{~cm} \mathrm{DBH,} \mathrm{and}$ Indian names and uses were recorded. In 0,1 hectare, 135 trees belonging to 60 species were found. Lecythidaceae, Leguminosae (sensu latu), Burseraceae, Lauraceae and Bombacaceae were the commonest families. Ninety-five percent of the species received a Waimiri Atroari name, and in a smller sample $(n=34)$, sixty-five percent of the species had some specific use. These results show a high degree of knowledge and utilization of the forest by the Waimiri Atroari.

Key Words: Waimiri Atroari, ethnobotany, floristics, Amazonia
\end{abstract}

(1) Trabalho financiado pelo ORSTOM/INPA e Pós-Graduação INPA/FUA

(2) Dept. de Engenharia Florestal, UTAM, Manaus - AM

(3) Pós-graduação em Ecologia-INPA, C.P.478, CEP 69000 Manaus - AM

(4) Convênio ORSTOM/INPA, Manaus - AM 


\section{Introduçāo}

Tradicionalmente, a metodologia usada em estudos etnobotânicos de grupos indígenas consiste em questioná-los sobre as espécies de plantas usadas na confecção de objetos, na alimentação, nos remédios, e nos rituais, conforme estas atividade são observadas pelo pesquisador. Nosso trabalho diverge desses métodos tradicionais e adota a metodologia usada por BALEE (1986), BOOM (1986) e PRANCE et al. (1987). Estes autores procuraram quantificar a utilidade da floresta para o indígena, determinando o número de espécies utilizadas em uma dada área de floresta como índice do grau de utilização da floresta. Esta metodologia consiste em definir uma área de floresta (no caso dos autores acima, 1 hectare) e coletar dentro da área amostras botânicas de todas as espécies de árvores. Posteriormente, são feitas entrevistas com os índios, onde cada amostra é identificada pelo seu nome indígena e são registradas informações sobre o uso da espécie. Além de quantificar o grau de utilização da floresta pelos indígenas, esta metodologia também pode fornecer dados florísticos, que podem ser de considerável importância, quando se leva em conta que muitas áreas indígenas estão em regiões pouco visitadas pelos botânicos.

A tribo Waimiri Atroari, do tronco linguístico Karib, tinha como território parte da margem esquerda do Rio Negro e alguns dos seus afluentes, mas devido a conflitos com os brancos por motivos de terra e principalmente a procura da castanha-do-Pará, no século passado e início deste século, recuaram para os afluentes Camanau, Curiaú, Alalau e Branquinho. Lá permaneceram com poucos contatos com os brancos até a década de 70 , quando foi iniciada a construção da BR-174 (Manaus - Caracaraí), cortando na metade o território dos Waimiri Atroari. Devido à resistência dos índios à abertura da estrada, muitos deles foram massacrados e aldeias dizimadas pelo lago da Usina Hidrelétrica de Balbina, e os seus moradores foram transferidos para novas aldeias, pelos Programa Waimiri Atroari (Convênio FUNAI/Eletronorte).

Hoje, existem 470 Waimiri Atroari, distribuídos em 11 aldeias. Apesar dos contactos históricos e atuais com os brancos, eles mantêm grande parte de sua cuitura intacta, como por exemplo, língua, rituais e sistema de produção agrícola. Este trabalho objetivou quantificar o conhecimento e utilização da floresta de terra firme pelos índios Waimiri Atroari, como levantamento preliminar para um estudo mais abrangente e de longo prazo.

\section{Área de Estudo}

Os dados obtidos neste trabalho foram obtidos na aldeia Maré, no Rio Camanau, localizado no município de Novo Airão - AM (Fig. 1). O levantamento foi feito ao longo de uma picada de caça, numa área distante aproximadamente $0,5 \mathrm{Km}$ da maloca. Esta área abrangeu um gradiente ecológico de floresta de terra firme para floresta de baixio com igarapé (curso d'água). A floresta é primária, porém faz parte da zona utilizada pelos indígenas para a retirada de produtos florestais para a alimentaçãı e como matéria-prima para artesanato, utensílios domésticos, remédios, etc. 


\section{Metodologia}

Foram demarcadas ao longo de uma picada de caça 5 parcelas de $10 \mathrm{x}$ $20 \mathrm{~m}$ (total $=0,1$ hectare) com intervalo de $15 \mathrm{~m}$ (Fig. 2). Nessas parcelas foram coletadas com podão amostras botânicas de todas as árvores acima de $6 \mathrm{~cm}$ de DAP, com exceção de alguns casos onde as árvores eram muito altas e não tinham acesso por cipós ou árvores vizinhas. As amostras foram identificadas e depositadas no herbário do Instituto Nacional de Pesquisas da Amazônia (INPA), Manaus. Nomes indígenas foram obtidos durante a coleta botânica. Informações sobre utilização foram obtidas durante a coleta e a noite com um grupo de índios. Devido a proibição de entrada de pesquisadores em áreas indígenas na Amazônia no ano de 1988, não foi possível obter informações de uso para todas as espécies durante o período do trabalho, e algumas informações adicionais foram obtidas posteriormente durante a visita de um dos membros da aldeia à FUNAI em Manaus.

Para avaliar a contribuição de cada espécie na composição florística utilizou-se o índice de importância das espécies (IVI. Tab. 1) que foi calculado seguindo o sistema utilizado por MORI et al. (1983) e BOOM (1986) como sendo a soma de frequência relativa, densidade relativa, e dominância relativa. Frequência relativa foi obtida contando o número de vezes que uma espécie ocorre em cada parcela e dividindo este número pelo total de ocorrências para todas as espécies em todas as parcelas. Densidade relativa é o número de árvores de uma espécie dividido pelo número total de árvores no levantamento (=135), e a dominância relativa é a área basal de uma espécie dividido pela área basal total para todas as árvores $\left(=62.189 \mathrm{~cm}^{2}\right)$.

A contribuição das famílias botânicas foi avaliada utilizando-se o índice de valor de importância da família (IVIF. MORI et al., 1983; BOOM, 1986) que é a soma das três porcentagens (diversidade relativa, densidade relativa, e dominância relativa) das espécies de cada família, multiplicada por 100 .

\section{Resultados e Discussōes}

\section{Florísticos}

As dez pesquisas mais importantes na área do levantamento são relacionadas na Tabela 1. A espécie mais importante foi Eschweilera coriacea (Lecythidaceae), que é uma das espécies mais comuns nas florestas da região de Manaus, sendo também frequente em outras partes da Amazônia (MORI, com. pes.).

O índice de valor de importância da família foi calculado para todas as famílias encontradas e as dez famílias mais importantes estão relacionadas na Tabela 2. As três famílias mais importantes foram as Lecythidaceae, Leguminosae (sensu latu, Burseraceae. Esta listagem pode ser considerada como normal dentro dos padrões florísticos das florestas da Amazônia Central.

A presença de Annonaceae, Meliaceae e Rubiaceae na $6^{\underline{a}}, 7^{\text {a }}$ e $9^{\mathbf{a}}$ posição respectivamente, entre as 10 famílias mais importantes, deve-se ao fato de ter- 
mos usado o DAP de $6 \mathrm{~cm}$ como limite mínimo neste estudo. Estas famílias contêm muitas espécies cujo ninho ecológico é o sub-bosque ou dossel inferior, que são dificilmente considerados em inventários que usam o DAP de $10 \mathrm{~cm}$, ou mesmo $30 \mathrm{~cm}$, no caso de inventários florestais comerciais. Por exemplo, a Annonaceae Duguetia flagellaris, que é uma espécie muito comum no sub-bosque da área de estudo, raramente ultrapassa um DAP de $10 \mathrm{~cm}$.

\section{Etnobotânicos}

Das 60 espécies coletadas no levantamento de 0,1 hectare, $95 \%$ foram identificadas com um nome específico na língua Waimiri Atroari, e as demais (4 espécies), receberam um nome geral ("wiwe") que quer dizer árvore.

Em uma amostra menor (34 espécies), $65 \%$ das espécies mostraram alguma utilidade para os índios (Tab. 3). Excluiu-se do conceito de espécies com utilidade aquelas que servem como lenha ou como fonte de alimento para os animais de caça, considerando-se apenas as que tinham alguma função específica. Possivelmente, o valor de $65 \%$ de utilização não reflete o real conhecimento dos índios sobre a floresta, pois metade das informações sobre o uso foram obtidas através de entrevistas com um membro jovem da tribo que visitou Manaus. Conforme completarmos o quadro de informações, este valor para a utilização da floresta poderá mudar.

\section{Conclusōes}

Os resultados obtidos para as 60 espécies coletadas (Conhecimento: 95\%, Utilização: $65 \%$ ) demonstraram um profundo conhecimerito da floresta pelos Waimiri Atroari e o papel fundamental da fioresta na sobrevivência deste povo. Se considerarmos que estes resultados são provenientes de uma amostra muito pequena de floresta, e que a floresta amazônica é floristicamente heterogênia, podemos esperar que tribos de regióes diferentes tenham conhecimentos específicos da flora dos seus territórios. Assim, a política vigente para a ocupação da Amazônia além de acarretar a degradação cultural de grupos indígenas está causando a perda de imensa riqueza de conhecimentos sobre a utilização da floresta. Tais conhecimentos são de utilidade não só para o índio, como também para o branco, podendo contribuir para um novo modelo de desenvolvimento da Amazônia.

\section{Referências Bibliograficas}

BALLE, W. 1986. Análise preliminar de inventário florestal e a etnobotânica ka'apor (Maranhão). Boletim do Museu Paraense Emilio Goeld, 2(2): 141-167.

BOOM, B. M. 1986. A forest inventory in Amazonian Bolivia. Biotropica 18(4):

187-294.

CARVALHO, J. P. F. de 1982. Waimiri Atroari a história não contada. Brasília. 180p.

MORI, S. A., A. M. de CARVALHO, \& T. S. dos SANTOS. 1983. Southern Bahian Moist Forests. Bot. Rev. 49(2): 155-232.

PRANCE, G. T., BALEE, W., BOOM, B. M. \& CARNEIRO, R. L. 1987. Quantitative Ethnobotany and the Case for Conservation in Amazonia, Conservation Biology, 1(4): 296-310. 


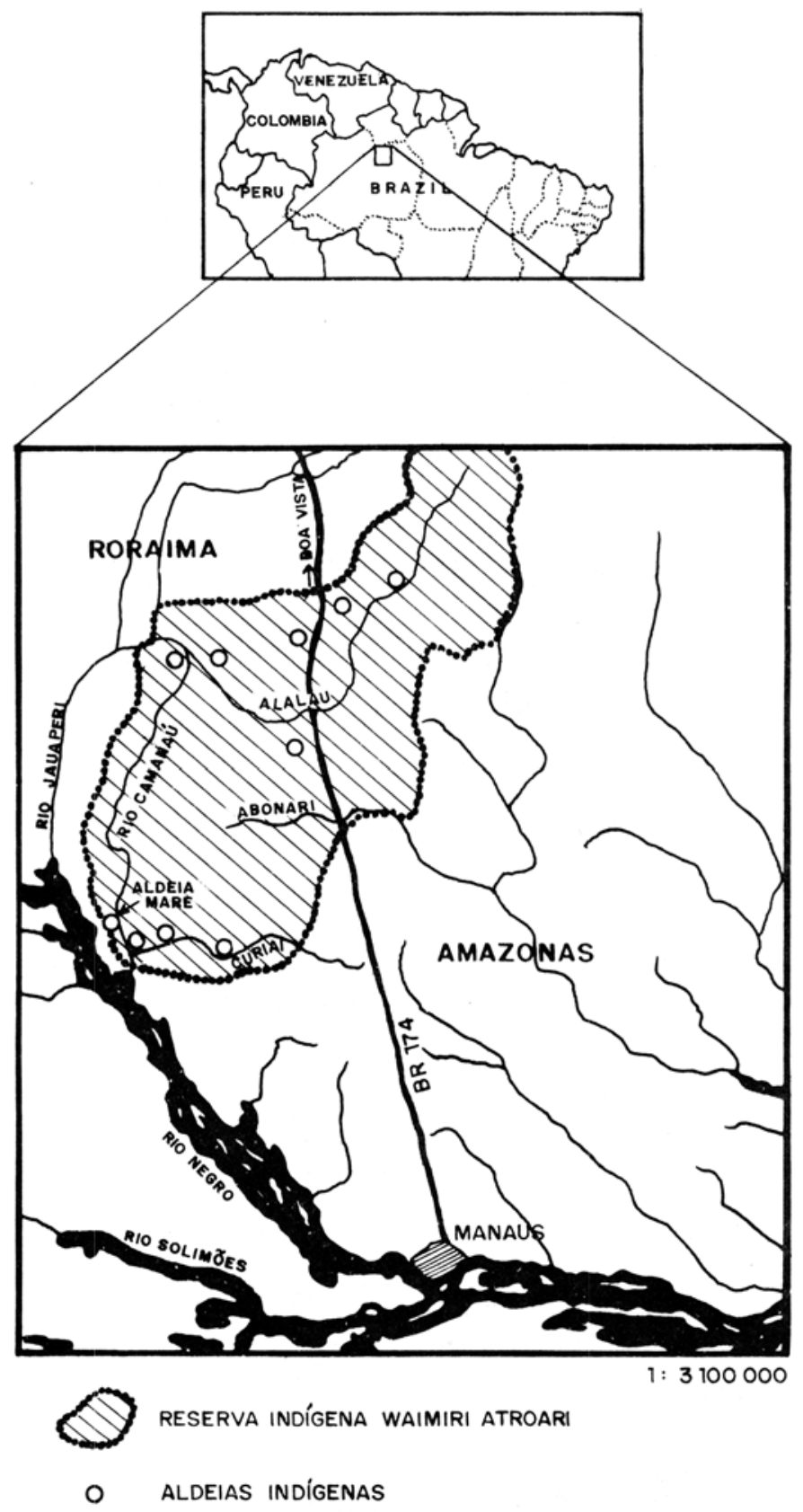

Figura 1 - Localização da reserva indígena Waimiri Atroari. 


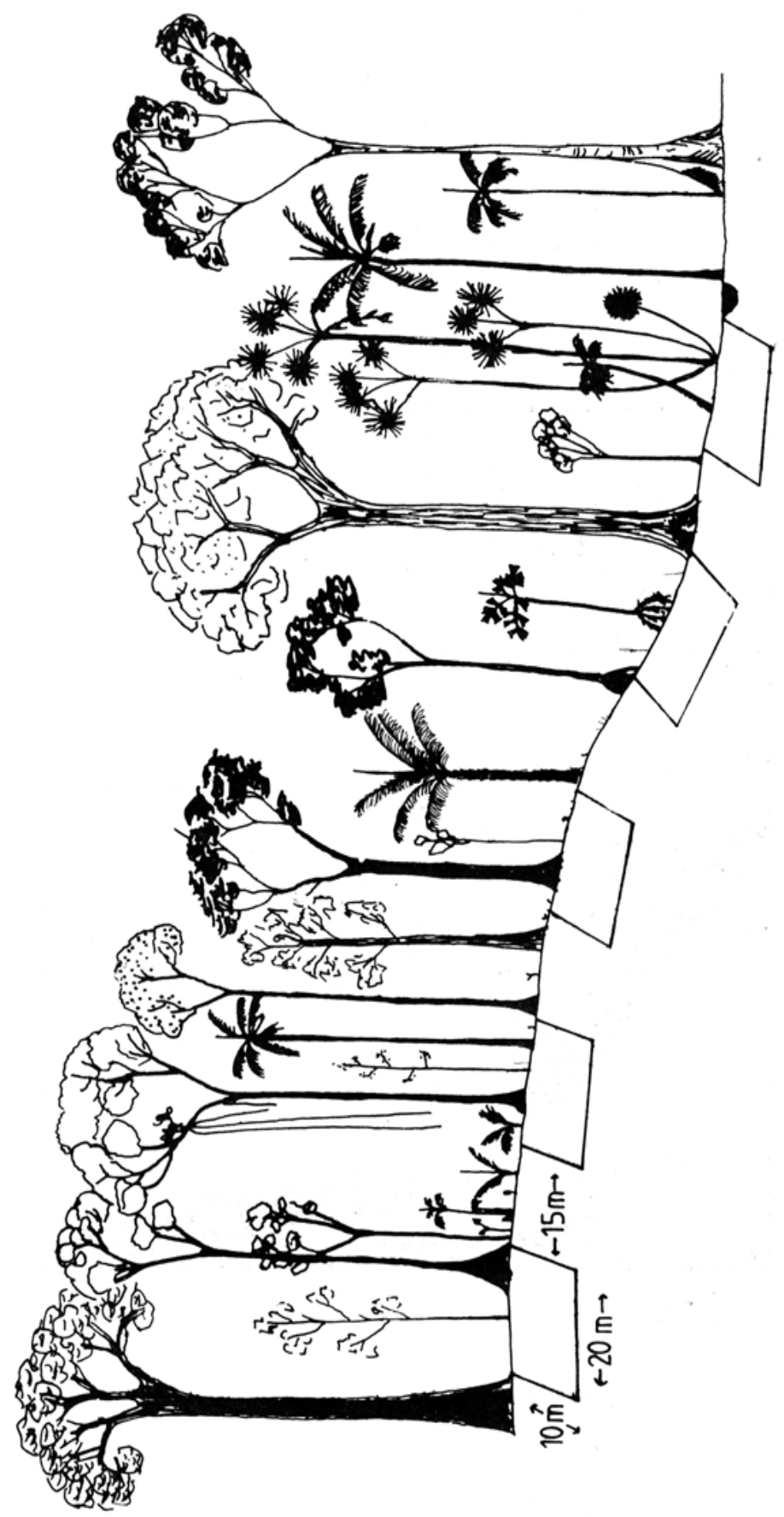

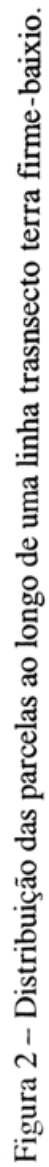


Tabela 1 - Espécies mais importantes numa amostra de 0,1 hectare de floresta Rio Camanau-AM

\section{ESPÉCIES}

Eschweilera coriacea

Scleronema micrantha

Protium grandifolium

Eschweilera sp.

Licaria guianensis

Goupia glabra

Clathrotropis macrocarpa

Licania oblongifolia

Macrolobium limabatum

Trichilia micrantha
(Lecythidac.)

(Bombacac.)

(Burserac.)

(Lecythidac.)

(Laurac.)

(Celastrac.)

(Leg. - Pap.)

(Chrysobalanac.)

(Leg. Caes.)

(Meliac.)
254,20

21,75

18,09

15,88

13,90

9,90

9,24

8.69

8,10

7,73

* Índice de Valor de Importância $(I V I)=$ Frequência relativa + Densidade relativa + Dominância relativa 
Tabela 2 - Famílias mais importantes numa amostra de 0,1 hectare de floresta Rio Camanau-AM

FAMÍLIAS

LECYTHIDACEAE

LEGUMINOSAE

BURSERACEAE

BOMBACACEAE

LAURACEAE

ANNONACEAE

MELIACEAE

CHRYSOBALANACEAE

RUBIACEAE

CELASTRACEAE

MORACEAE

\section{$I V I F^{*}$}

64,9

25,6

24.0

23,0

19,8

15,0

13,8

13,2

12,1

99,9

9,9

* Índice de Valor de Importância Familial (IVIF) $=$ Diversidade relativa + Densidade relativa + Dominância relativa 
Conhecimento e utilizaçảo da floresta...

Tabela 3 - Espécies de árvores utilizadas pelo Waimiri Atroari, em amostra de 0,1 hectare Rio Camanau-AM

ÁRVORE

PARTE USADA

USO

\section{ANNONACEAE}

Bocageopsis multiflora

galho com folhas

- maraca na dança

Duguetia flagellaris

indivíduo jovem

- caniço

fruto

- comestível

Indeterminada

fibra do cortex

- alças p/ jamaxí

BOMBACACEAE

Scleronema micrantha

fibra do cortex

- alças p/ jamaxí

\section{BURSERACEAE}

Protium granifolium

madeira

- utensílio $\mathrm{p} / \mathrm{fazer}$ fogo

Protium altroni

madeira

- utensílio p/ fazer fogo

breu

- queimado p/ fazer

tinta $\mathrm{p} /$ artesanato

CHRYSOBALANACEAE

Licania longistyla

casca

- queimada e misturada

c/ barro p/ fortalecer cerâmica

\section{CLUSIACEAE}

Symphonia globulifera látex

- pintura corporal e fixar apenas na flecha 
LAURACEAE

Nectandra?

tronco

- canoa

LECYTHIDACEAE

Eschweilera coriacea

fibra do cortex

- alças p/ jamaxí

LEG. CAESALPINIOIDAE

Hymenaea sp.

resina

- envernizar cerâmica

LEG. MIMOSOIDAE

Inga sp.

fruto

- comestível

LEG. PAPILIONOIDAE

Clathrotropis macrocarpa semente

- farinha comestível

MELASTOMATACEAE

Miconia serialis

fruto

- comestível

\section{MELIACEAE}

Trichilia micrantha

casca

- remédio $\mathrm{p} /$ picada

de tocandeira

MENISPERMACEAE

Abuta grandifolia

fruto

- comestível

MORACEAE

Pseudolmedia laevigata fruto

- comestível

RUBIACEAE

Chimarris barbata

fruto

- comestível

STECULIACEAE

Theobroma sp.

fruto

- comestível 